\title{
Hypermethylation and downregulation of glutathione peroxidase 3 are related to pathogenesis of melanoma
}

\author{
HAIYUE CHEN $^{1 *}$, ZHENLONG ZHENG $^{2 *}$, KI-YEOL KIM $^{3 *}$, XUEMEI JIN ${ }^{1}$, MI RYUNG ROH ${ }^{4}$ and ZHEHU JIN ${ }^{2}$ \\ Departments of ${ }^{1}$ Pathology, ${ }^{2}$ Dermatology, Yanbian University Hospital, Yanji City, Jilin, P.R. China; \\ ${ }^{3}$ BK21 PLUS Project, Yonsei University College of Dentistry; ${ }^{4}$ Department of Dermatology and Cutaneous \\ Biology Research Institute, Yonsei University College of Medicine, Seoul, Republic of Korea
}

Received March 31, 2016; Accepted August 8, 2016

DOI: $10.3892 /$ or.2016.5071

\begin{abstract}
As a crucial antioxidant enzyme, glutathione peroxidase 3 (GPX3) has been found to be frequently repressed in many cancers due to promoter hypermethylation and is known as a possible tumor suppressor gene. In the present study, we investigated whether promoter hypermethylation of GPX3 and its repression are present in melanoma and, if so, whether GPX3 downregulation is implicated in the pathogenesis of melanoma. Our results revealed methylation of GPX3 and downregulation of its expression in both melanoma cell lines and surgical melanoma tissue samples. In melanoma cell lines, GPX3 expression was restored by treatment with 5-aza-2'-deoxycytidine both in mRNA and protein levels. Depletion of GPX3 was found to increase the proliferative ability, motility, and invasiveness of melanoma cells. Moreover, negative expression of GPX3 was related to poor prognosis in melanoma patients. These results suggest that methylation-mediated GPX3 repression may have critical implications for melanoma pathogenesis.
\end{abstract}

\section{Introduction}

As well known potential carcinogens, reactive oxygen species (ROS) are involved in both carcinogenesis and tumor progression via ROS-induced DNA damage (1-4). During normal metabolism, cells avoid oxidative damage by means of antioxidant defense mechanisms that control the balance between the generation and removal of oxygen radicals. The anti-

Correspondence to: Professor Zhehu Jin, Department of Dermatology, Yanbian University Hospital, Yanji City, Jilin 133000, P.R. China

E-mail: jinzh_621@163.com

Professor Mi Ryung Roh, Department of Dermatology, Yonsei University College of Medicine, Gangnam Severance Hospital, 211 Eonjuro, Gangnam-gu, Seoul 135-720, Republic of Korea E-mail: karenroh@yuhs.ac

${ }^{*}$ Contributed equally

Key words: GPX3, promoter hypermethylation, melanoma oxidant system includes various enzymes, such as superoxide dismutase and catalase, as well as glutathione peroxidase (GPX) (5,6).

GPX3, a member of the GPX family, is an extracellular glycosylated enzyme that reduces both phospholipid hydroperoxides and fatty acid hydroperoxides using various electron donors, such as glutathione, thioredoxin, and glutaredoxin (7-10). It is generally known that hydroperoxides are involved in various biological behavior of cancer cells such as proliferation, motility, and invasion (11). Therefore, some investigators expected that GPX3 might play a role in cancer carcinogenesis by controlling the hydroperoxide levels inside cells (11).

The human GPX3 gene, located on chromosome 5q32, is the only known extracellular antioxidant isoform containing selenocysteine at the catalytic site (12-15). GPX3 activity is regulated by the availability of selenium, a well known cancer chemopreventive agent (16). Selenium uptake is related to decreased cancer incidence, and although there is no direct experimental evidence, this linkage may be related to the anticancer effect of selenium (17).

GPX3 is mainly synthesized by the proximal tubules of the kidney (18). GPX3 is readily detectable in extracellular body fluids such as the aqueous humor in the anterior and posterior chambers of the eye, blood plasma, and thyroid colloid $(19,20)$. Moreover, transcripts of GPX3 are also detected in epithelial cells of the oviduct (21). The mechanisms that regulate the transcription of GPX3 remain largely unknown. Recent studies have shown that oxidative stress can induce transcriptional upregulation of GPX3 in inflammatory bowel disease and experimental colitis, in patients with asthma, and in the diabetic mouse heart (22-25). However, GPX3 expression is usually downregulated in cancer tissues compared to its expression in normal tissues (26). In contrast, overexpression of GPX3 in prostate cancer cells has been found to attenuate tumorigenic potential and spontaneous metastasis in vivo (27). $G P X 3$ may serve as a potential tumor suppressor gene in cancer progression.

Genetic and epigenetic mechanisms lead to inactivation of tumor suppressor genes in cancers. As one of the major modifications involved in epigenetic inactivation of genes, abnormal methylation in $\mathrm{CpG}$-rich promoter regions can induce transcriptional silencing of tumor suppressor genes. Promoter hypermethylation of GPX3 is frequently detected in 
various cancer tissues, such as gastric, prostate, and Barrett's esophageal cancer, and this may be a common and crucial cause of GPX3 downregulation in cancers.

Malignant melanoma is a common leading cause of cancerrelated death among patients with skin cancer. The incidence of cutaneous melanoma has rapidly increased over the last 50 years (28). However, the molecular basis of melanoma is largely unknown. In the current study, for the first time, we determined whether GPX3 is also downregulated in melanoma and, if so, whether promoter hypermethylation is associated with its repression. We further investigated the influence of GPX3 on the biological behavior of melanoma cells and its clinicopathological significance in melanoma patients.

\section{Materials and methods}

Patients. Two tissue microarray (TMA) sections of melanoma samples were purchased from Pantomics, Inc. (Richmond, CA, USA) and Super BioChips (Seoul, Korea), respectively, and used for immunohistochemistry. The specimens included 70 primary melanoma and 25 metastatic melanoma tissue samples. Three normal skin tissues and 10 primary melanoma tissue samples were obtained from Yanbian University Hospital and used for quantitative real-time PCR (qRT-PCR) and methylation-specific PCR (MSP) analysis. The clinicopathological characteristics of all patients are shown in Table I. This study was approved by the Institutional Review Board of Yanbian University Hospital.

Cell culture and establishment of GPX3 knockdown $S K$-MEL-24 cells. The human melanoma cell lines (SK-MEL-2 and SK-MEL-24), and adult human epidermal melanocytes (HEMs) were purchased from ATCC (Rockville, MD, USA). Melanoma cell lines were cultured in microvascular endothelial cell medium (MEM) supplemented with $10 \%$ fetal bovine serum (FBS), $100 \mathrm{U} / \mathrm{ml}$ ampicillin, $100 \mu \mathrm{g} / \mathrm{ml}$ streptomycin, and $2 \mathrm{mM}$ glutamine (Gibco BRL, Gaithersburg, MD, USA). HEMs were cultured in $\mathrm{MGM}^{\mathrm{TM}}-4$ melanocyte cell basal medium (Lonza Group AG, Basel, Switzerland) supplemented with MBM-4 plus SingleQuots ${ }^{\mathrm{TM}}$. Cells were grown in a humidified atmosphere of $5 \% \mathrm{CO}_{2}$ at $37^{\circ} \mathrm{C}$. SK-MEL-24 cells were positive for GPX3 on both the mRNA and protein levels. GPX3-depleted stable SK-MEL-24 cell lines were created using GPX3 shRNA-encoding plasmid (Origin Pharmaceutical Services Ltd., Abingdon, Oxfordshire, UK). Retroviruses were generated in 293T cells and the transduced cells were selected with $1 \mu \mathrm{g} / \mathrm{ml}$ puromycin. Silencing of GPX3 in SK-MEL-24 cell lines was confirmed by reverse transcription polymerase chain reaction (RT-PCR) analysis (Fig. 1A).

Immunofluorescence in TMA sections. Melanoma tissue sections were deparaffinized in xylene and rehydrated in graded alcohol. Antigen retrieval was performed by a 2 min autoclave treatment using antigen retrieval buffer (Dako North America, Inc., Carpinteria, CA, USA). Sections were treated with primary antibody against GPX3 (working dilution: 1:100; Abcam plc, Cambridge, UK) at room temperature (RT) for $1 \mathrm{~h}$, and the Donkey Anti-Mouse IgG H\&L (Alexa Fluor ${ }^{\circledR} 488$ ) (Abcam) was used as the secondary antibody. Counterstaining was performed using 4',6-diamidino-2-phenylindole (DAPI).
Table I. Characteristics of the patients with malignant melanoma.

\begin{tabular}{lcc}
\hline & \multicolumn{2}{c}{ No. of patients $(\%)$} \\
\cline { 2 - 3 } Variables & Primary melanoma & Metastatic melanoma \\
\hline Total cases & 80 & 25 \\
Age, years & & $55(25-73)$ \\
Median age (range) & $56(11-84)$ & \\
Gender & & $14(56.0)$ \\
Male & $47(58.8)$ & $11(44.0)$ \\
Female & $33(41.2)$ & $4(16.0)$ \\
Survival & & $9(36.0)$ \\
Alive & $12(15.0)$ & $12(48.0)$ \\
Dead & $34(42.5)$ & \\
Unknown & $34(42.5)$ & \\
\hline
\end{tabular}

In this study, sections of cell blocks from HeLa cells were used as a positive control for staining of GPX3. Rabbit IgG (R\&D Systems, Wiesbaden-Nordenstadt, Germany) was used as a negative control in this study.

Immunocytochemistry in human melanoma cell lines and adult HEMs. GPX3 expression was determined in both HEMs and melanoma cell lines by immunocytochemistry. Cell lines were plated on glass coverslips containing 6-well plates on the day before staining. After fixation with $95 \%$ ethanol, the cells were blocked with $5 \%$ bovine serum antigen (BSA) at RT for $1 \mathrm{~h}$. Endogenous peroxidase activity was inactivated with a mixture of $\mathrm{H}_{2} \mathrm{O}_{2}$ and methanol, in a ratio of $1: 40$. The cells were then treated with primary antibody against GPX3 (working dilution: 1:200; Abcam) at RT for $1 \mathrm{~h}$, and the Real $^{\mathrm{TM}}{ }^{\mathrm{EnV}}$ ision $^{\mathrm{TM}}$ HRP Rabbit/Mouse detection system (Dako North America, Inc.) was used as the secondary antibody. After visualization with 3,3'-diaminobenzidine, counterstaining was performed using hematoxylin. HeLa cells were used as a positive control for GPX3.

Total RNA extraction and analysis for GPX3 mRNA expression. Total RNA was isolated with TRIzol reagent (for cell line samples; Invitrogen, Carlsbad, CA, USA) and the RNeasy FFPE kit (from paraffin tissue samples; Qiagen $\mathrm{GmbH}$, Hilden, Germany), and cDNA was synthesized using total RNA and AccuPower ${ }^{\circledR}$ RT PreMix (Bioneer, Seoul, Korea) according to the manufacturer's protocol. qRT-PCR was performed as previously described using 1X SYBR-Green Master Mix (Applied Biosystems, Foster City, CA, USA) and synthesized cDNA in an ABI7500 Real-time PCR system (Applied Biosystems) under the following conditions: initial denaturation for $10 \mathrm{~min}$ at $95^{\circ} \mathrm{C}$, followed by 40 cycles of $95^{\circ} \mathrm{C}$ for $20 \mathrm{sec}, 50^{\circ} \mathrm{C}$ for $30 \mathrm{sec}$, and $72^{\circ} \mathrm{C}$ for $45 \mathrm{sec}$. Conventional RT-PCR analysis was performed using AccuPower PCRPreMix (Bioneer) and the synthesized cDNA with an annealing temperature of $58^{\circ} \mathrm{C}$. $\beta$-actin was used as the housekeeping gene. Oligonucleotide primers used for the PCR were: 5'-CAACCAATTTGGAAAACAGG-3' and 5'-GTG 
A

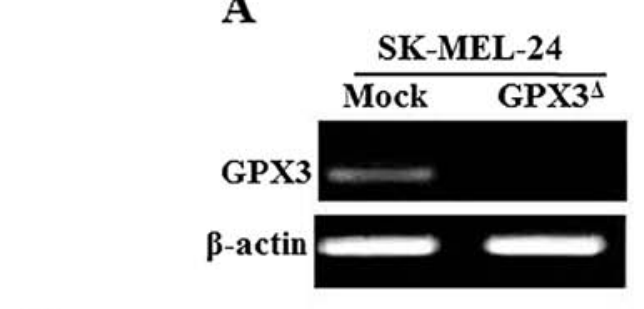

B

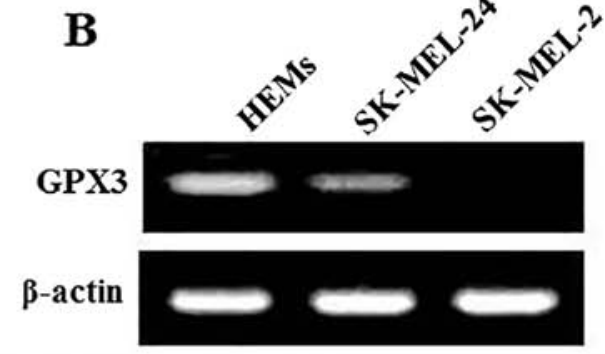

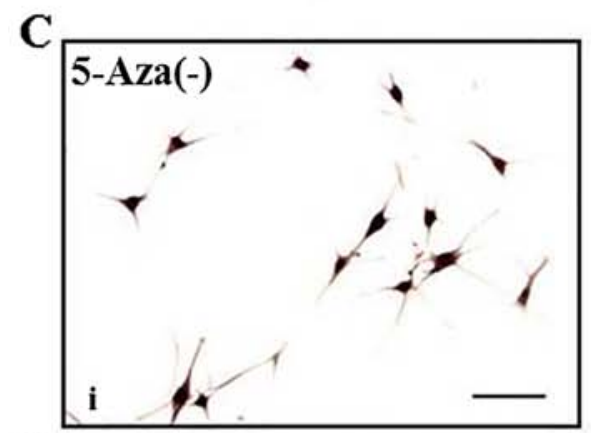
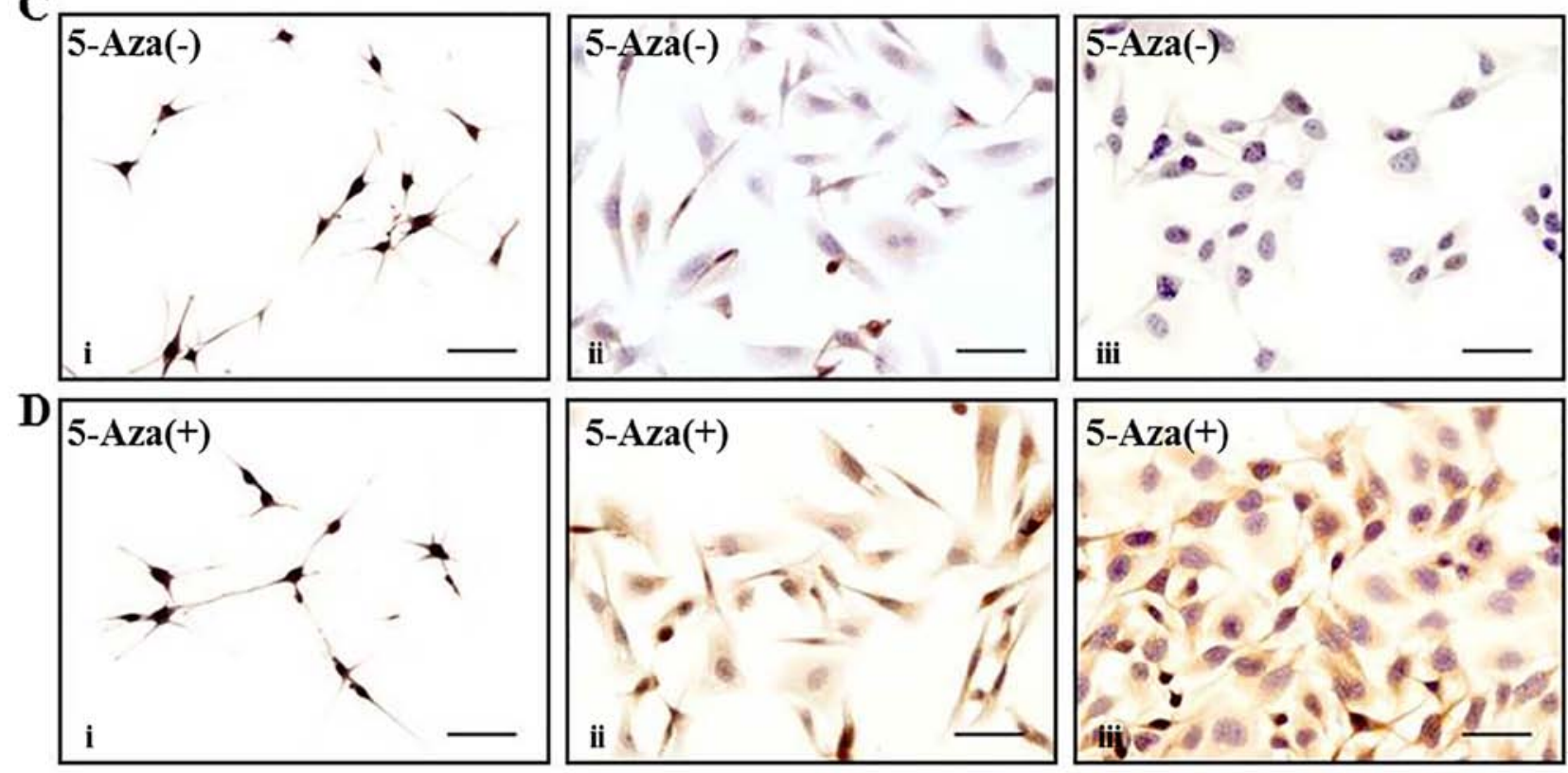

$\mathbf{E}$

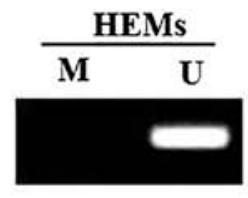

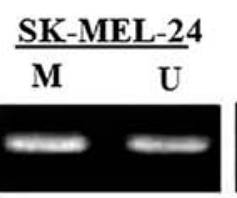

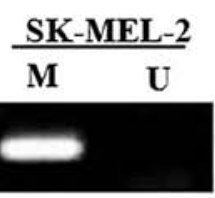

$\mathbf{F}$

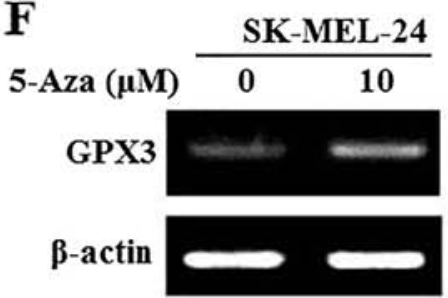

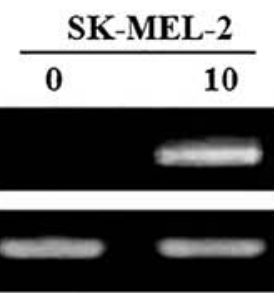

Figure 1. Depletion of GPX3 using retroviral vector-based shRNA. The mRNA (A) expression was analyzed in SK-MEL-24 Mock and GPX3 ${ }^{\Delta}$ cells. The mRNA (B) and protein (C) expression of GPX3 were detected in HEMs (i), SK-MEL-24 (ii), and SK-MEL-2 (iii) cells. GPX3 expression was decreased in melanoma cell lines compared to HEMs both in mRNA and protein levels. Promoter methylation of GPX3 was found in melanoma cell lines (E) and both protein (D) and mRNA (F) expression were restored by 5-Aza treatment.

GGAGGACAGGAGTTCTT-3' for GPX3; and 5'-ATAGC ACAGCCTGGATAGCAACGTAC-3' and 5'-CACCTTC TACAATGAGCTGCGTGTG-3' for $\beta$-actin (29).

Treatments with 5-aza-2'-deoxycytidine (5-Aza). Two melanoma cell lines (SK-MEL-24 and SK-MEL-2) were treated with 5-Aza (Sigma-Aldrich, Inc., St. Louis, MO, USA), 1 or $10 \mu \mathrm{M}$, for $72 \mathrm{~h}$, with drug replacement every $24 \mathrm{~h}$. Cells were then harvested and mRNA expression of GPX3 was detected in each group of the cells.

DNA extraction and MSP. Genomic DNA extraction was performed in HEMs and melanoma cell lines, three normal skin samples, and 10 melanoma tissue samples using the QIAamp DNA mini kit (Qiagen $\mathrm{GmbH}$ ). Modification of DNA was performed with the EZ DNA Methylation kit (Zymo
Research Corp., Irvine, CA, USA), and the methylationspecific or unmethylation-specific amplification was performed with an annealing temperature of $59^{\circ} \mathrm{C}$ using the following primers: 5'-TATGTTATTGTCGTTTCGGGAC-3' and 5'-GTCCGTCTAAAATATCCGACG-3' for methylationspecific amplification; and 5'-TTTATGTTATTGTTT TGGGATG-3' and 5'-ATCCATCTAAAATATCCAACAC TCC-3' for unmethylation-specific amplification. The amplified DNA products were loaded on $2 \%$ agarose gels for electrophoresis and stained with ethidium bromide.

Cell proliferation assay. To determine the influence of GPX3 on the proliferative ability of the melanoma cells, the trypan blue exclusion assay was performed in GPX3-depleted SK-MEL-24 $\left(\right.$ GPX $\left.^{\Delta}\right)$ cells and cells transfected with the control vector (Mock). Cells were seeded in 6-well plates and 
A

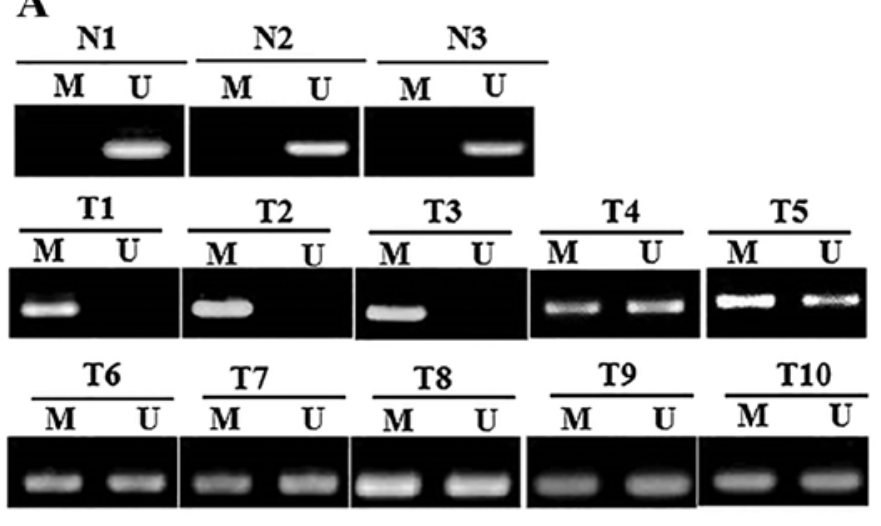

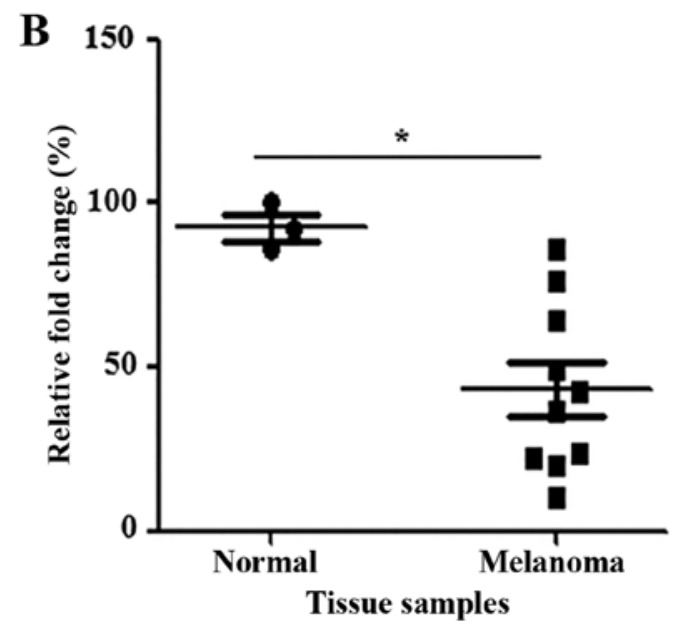

Figure 2. Promoter methylation of GPX3 was detected more often in melanoma tissues than normal skin tissues (A); The mRNA expression of GPX3 was significantly decreased in primary melanoma tissues than normal skin tissues (B). The results were analyzed by the Mann-Whitney $\mathrm{U}$ test $\left({ }^{*} \mathrm{P}=0.018\right)$.

the number of cells in each group was counted at 36 and $72 \mathrm{~h}$ using a hemocytometer.

Cell migration assay. To evaluate the influence of GPX3 on the migration ability of the melanoma cells, a wound healing assay was performed in GPX $3^{-}$- and Mock-SK-MEL-24 cells. The cells were seeded in 24-well culture plates in triplicate. After $24 \mathrm{~h}$ of culturing, linear scrape injuries were made with a yellow pipette tip on the cells that displayed growth to a confluent monolayer. Wound closing in each group was photographed at the indicated time points and analyzed with the Image $\mathbf{J}$ analysis software package (National Institutes of Health, Bethesda, MD, USA).

Cell invasion assay. To evaluate the influence of GPX3 on the invasiveness of the melanoma cells, an invasion assay was performed using Transwell filters (pore size, $8 \mu \mathrm{m}$; BD Biosciences, Bedford, MA, USA) in GPX3 ${ }^{-}$- and MockSK-MEL-24 cells. Both the lower and top sides of Transwell filters were coated with $8 \mu \mathrm{g} / \mu$ l Matrigel (BD Biosciences, San Jose, CA, USA) and the filters were placed on 24-well culture plates with a culture medium with $15 \%$ BSA. The cells were suspended in a medium containing 3\% BSA and then seeded in the coated Transwell chambers. After $36 \mathrm{~h}$ of culturing, the bottom side of the membrane with the invaded cells was fixed with $4 \%$ paraformaldehyde and then stained with crystal violet. The number of invaded cells was counted with a microscope and comparatively investigated between GPX $3^{4}$ - and Mock-SK-MEL-24 cells.

Statistical analysis. In this study, we used commercially available software (SPSS version 15.0 for Windows; SPSS, Inc., Chicago, IL, USA) for statistical analysis. The Mann-Whitney $\mathrm{U}$ test was used to analyze differences between groups in the in vitro studies. The $\chi^{2}$ test and Fisher's exact test were used to investigate differences between groups of clinical samples. Survival analysis for melanoma patients was performed using Kaplan-Meier analysis, and the differences were compared using the log-rank test. A value of $\mathrm{P}<0.05$ was considered to indicate a statistically significant difference.

\section{Results}

GPX3 is downregulated in melanoma cell lines by promoter hypermethylation. In the current study, both mRNA and protein expression of GPX3 were comparatively investigated in HEMs and melanoma cell lines. Our results showed that GPX3 expression was downregulated in melanoma cell lines to a greater extent than in HEMs on both the mRNA and protein levels (Fig. 1B and C). The methylation status of $G P X 3$ was evaluated in both HEMs and melanoma cell lines using MSP primers against the GPX3 promoter. Full methylation (presence of only methylated $\mathrm{CpGs}$ ) was found in the SK-MEL-2 cell line, which was negative for GPX3 on both the RNA and protein levels. Moreover, HEMs and SK-MEL-24 cells, with expression of GPX3 on both the RNA and protein levels, showed unmethylation (presence of only unmethylated CpGs) and partial methlylation (presence of both methylated and unmethylated CpGs) for GPX3, respectively (Fig. 1E). To investigate whether methylation status was related to repression of GPX3 expression, melanoma cell lines were treated with the DNA demethylation agent 5-Aza, and our results showed that demethylation of the GPX3 gene by 5-Aza in both SK-MEL-2 and SK-MEL-24 cell lines restored GPX3 expression at mRNA and protein levels (Fig. 1D and F). No change was found in GPX3 expression of HEMs according to the presence or absence of 5-Aza treatment (Fig. 1D).

GPX3 promoter is methylated in primary melanoma tissues. The methylation status of GPX3 was also determined in 3 normal skin and 10 primary melanoma tissue samples. Unmethylation for GPX3 was found in the 3 normal skin samples. By contrast, full methylation and partial methylation were detected in three and seven melanoma tissues samples, respectively (Fig. 2A). Moreover, RT-PCR analysis showed that GPX3 mRNA expression was significantly decreased in primary melanoma tissues compared to normal skin tissues (Fig. 2B).

The influence of GPX3 downregulation on the biological behavior of SK-MEL-24. SK-MEL-2 showed increased 
A

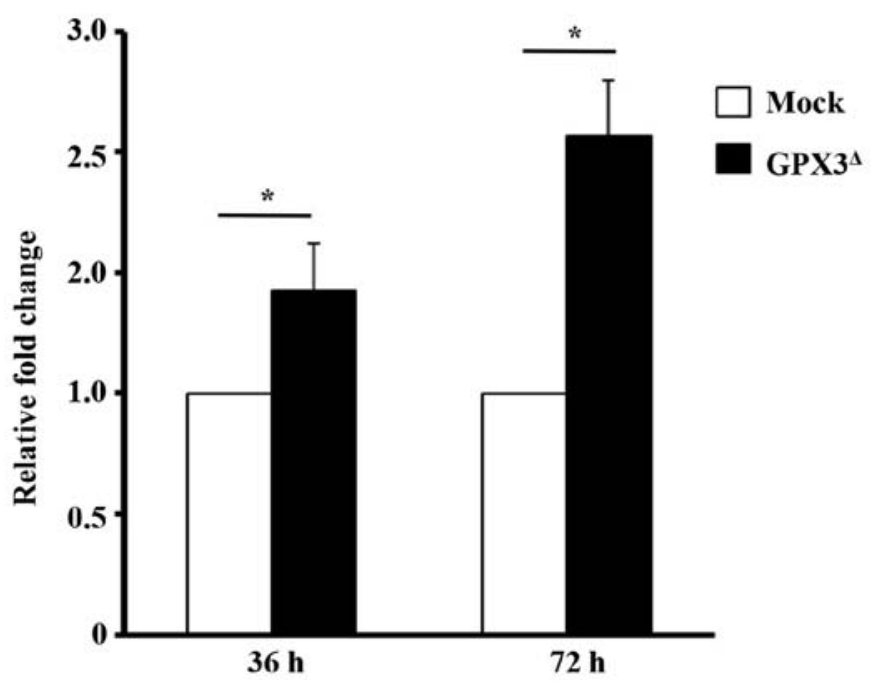

C

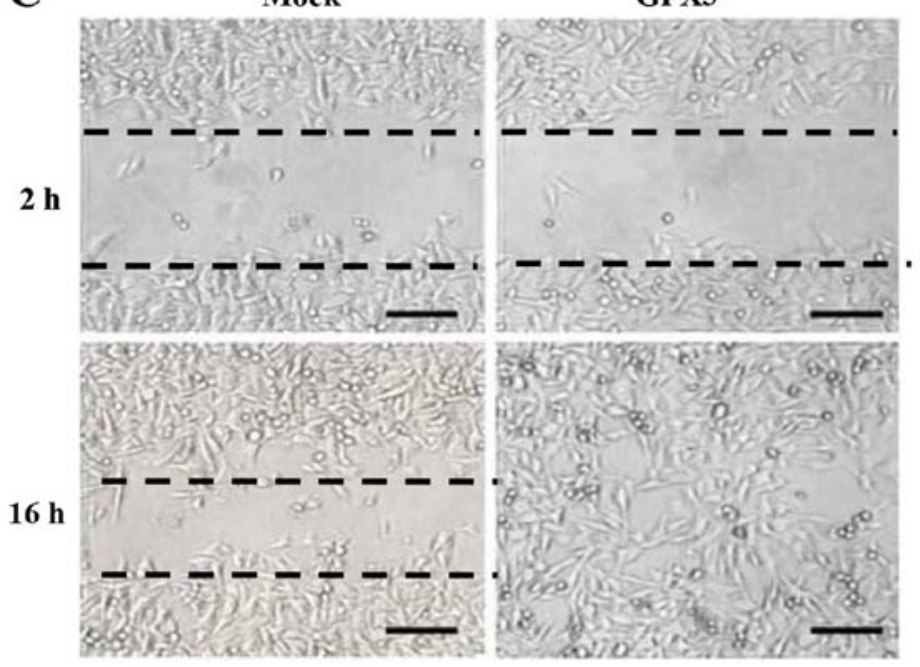

B i $^{2}$

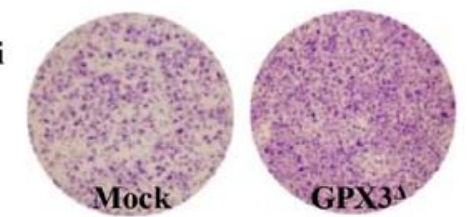

ii

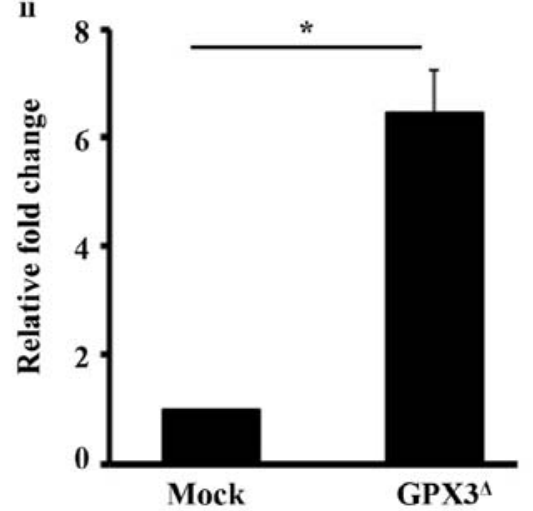

D

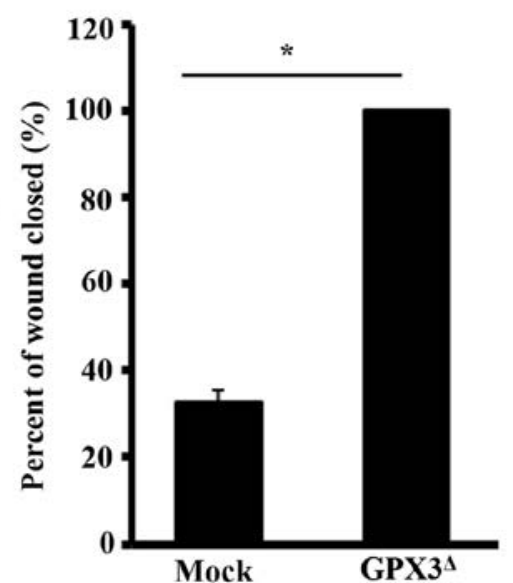

Figure 3. The influence of GPX3 downregulation on the biological behavior of SK-MEL-24. The proliferative (A), invasive (B), and migration (C and D) ability were significantly increased in GPX3 depleted SK-MEL-24 cells compared to Mock-SK-MEL-24 cells. The results are shown as mean values \pm SD and were analyzed by the Mann-Whitney U test ( $\left.{ }^{*} \mathrm{P}<0.001\right)$.

invasion ability than SK-MEL-24 cells, which was found to be negative for GPX3 on both RNA and protein levels (data not shown). However, SK-MEL-2 and SK-MEL-24 cells may have a different molecular basis. Therefore, the differences in invasive ability between the cells cannot exactly represent the role of GPX3. We also found that invasive activities were suppressed to a greater extent in SK-MEL-2 and SK-MEL-24 cells with 5-Aza treatment than without 5-Aza treatment (data not shown). However, no change was found in HEMs according to the presence or absence of 5-Aza treatment. Other tumor suppressor genes in addition to GPX3 have been found to be methylated in melanoma (30). Consequently, 5-Aza treatment can restore the RNA and protein expression of various genes that are methylated in melanoma. Therefore, we constructed GPX3-depleted cell lines to investigate the influence of GPX3 on the biological behavior of melanoma cells.

The proliferative, migration, and invasive ability of cells were comparatively investigated in the GPX $3^{\Delta}$ - and Mock-
SK-MEL-24cells. Compared to Mock-SK-MEL-24 cells, GPX $3^{\Delta}$-SK-MEL-24 cells demonstrated 1.43- and 2.07-fold higher proliferative ability in each time point, respectively $\left(\mathrm{P}<0.001\right.$, and $\mathrm{P}<0.001$, respectively). Moreover, GPX3 ${ }^{{ }_{-}}$ SK-MEL-24 cells showed 3.06-fold $(\mathrm{P}<0.001)$, and 6.47-fold $(\mathrm{P}<0.001)$ higher migration and invasive ability, respectively (Fig. 3A-D).

Clinicopathological significance of GPX3 expression in melanoma patients. GPX3 expression was detected in the cytoplasm of cancer cells in melanoma tissue samples (Fig. 4A). The expression frequency of GPX3 was significantly increased in primary sites $(33.8 \%)$ compared to metastatic sites $(10.8 \%)$ of melanoma tissues $(\mathrm{P}=0.036)$ (Fig. 4B). Kaplan-Meier analysis was performed in 46 primary malignant melanoma patients. We found that patients with GPX3 expression showed increased survival rates (median survival duration 71 months) compared to patients without GPX3 expression (median survival dura- 
A
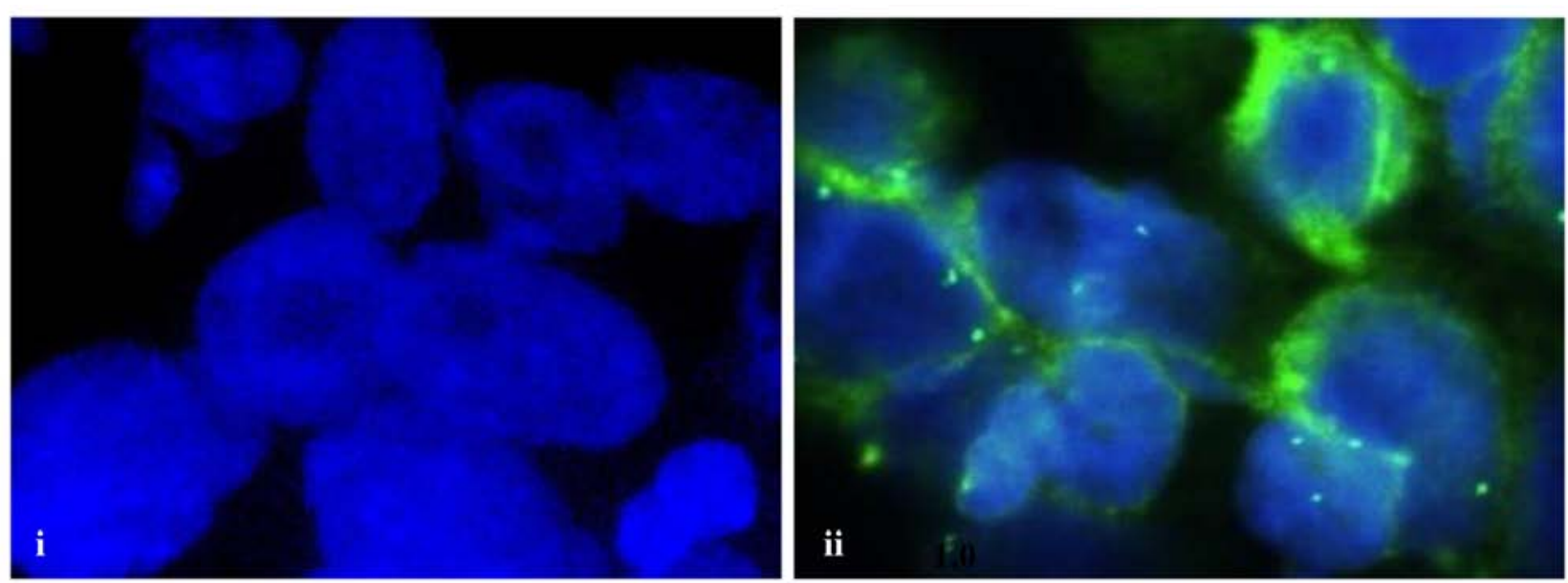

B

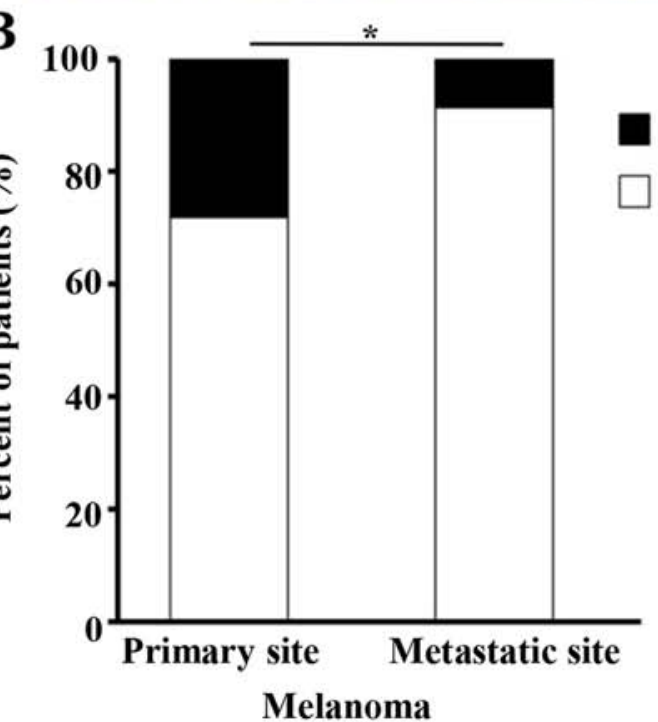

C

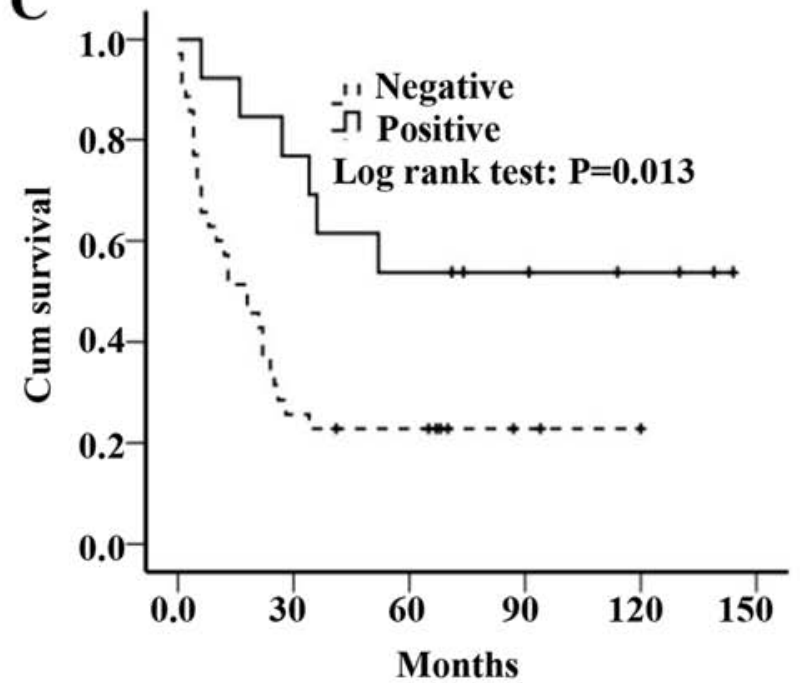

Figure 4. Clinicopathological significance of GPX3 expression in melanoma patients: Negative (i) and positive (ii) patterns of GPX3 expression in melanoma tissues (A). GPX3 expression was found in cytoplasm of cancer cells in melanoma tissues (i), and was detected more often in primary site than metastatic site (B). The results were analyzed by $\chi^{2}$-test ( $\mathrm{P}=0.036$ ). According to the Kaplan-Meier analysis, negative GPX3 expression significantly related to poor prognosis of melanoma patients $(\mathrm{C})$.

tion 18 months) $(\mathrm{P}=0.013)$ (Fig. 4C). No significant association was found between GPX 3 expression and age or gender of melanoma patients.

\section{Discussion}

The antioxidant system is the first line of defense against ROS-induced damage. GPX3, a major antioxidative enzyme, is known as a critical scavenger of ROS. Silencing of GPX3 promotes ROS production in muscle stem cells and colon cancer cell lines $(31,32)$. GPX3 is selectively expressed in several healthy tissues, such as the esophagus, stomach, and colon (26). In the current study, GPX3 expression was detected in the cytoplasm of HEMs, but decreased or negative expression was found in melanoma cell lines. Moreover, unmethylation was found in HEMs, while full or partial methylation was observed in melanoma cell lines. Methylation of GPX3 was also frequently detected in the human melanoma tissue samples, and mRNA expression was concurrently repressed in melanoma tissue samples, in the present study. Supportively, 5-Aza treatment can restore the expression of GPX3 in mela- noma cell lines. 5-Aza inhibits DNA-methyltransferase activity and thereby restores the expression of silenced genes (33). Downregulation and silencing of GPX3 in melanoma may result from promoter hypermethylation.

Many studies have shown that GPX3 is frequently methylated and downregulated in cancers compared to related normal tissues, and it has been hypothesized that GPX3 may act as a tumor suppressor in cancers. However, the effect of GPX3 on the biologic behavior of cancer cells has not been fully investigated (11). Some investigators showed that GPX3 overexpression can promote cancer cell motility but not the growth of cancer cells in gastric cancer (34). In prostate cancer cell lines, GPX3 overexpression inhibits the invasiveness of cells in vitro, and can reduce tumor volume and prevent metastasis in vivo (27). In the current study, we found that GPX3 downregulation can promote the proliferation, motility, and invasion of melanoma cells in vitro. In support of these observations, negative expression of GPX3 was more frequently detected in metastatic site than primary site of melanoma and was also found to be a negative prognostic indicator for melanoma patients in the present study. The clinical implications 
of GPX3 have also been mentioned by other investigators. In ovarian clear cell adenocarcinoma, GPX3 expression is related to cisplatin sensitivity in patients. Moreover, downregulation and promoter methylation of GPX3 were detected more often in gastric cancer patients with lymph node metastasis. Downregulation of GPX3 was also related to poor prognosis in patients with various cancers such as multiple myeloma, gastric cancer, and gallbladder cancer (35-37). Loss of expression of Nkx3.1, a well known tumor suppressor gene in prostate cancer, can induce increased oxidative damage in prostate carcinogenesis. In Nkx3.1 knockout mice, GPX3 expression strongly increased during conversion from normal prostate epithelium to prostatic intraepithelial neoplasia (PIN), and this expression is mainly found in tumor cells of PIN lesions. However, the loss of PTEN that is induced by increased oxidative damage can decrease GPX3 expression during the transformation from PIN to cancer in Nkx3.1 knockout mice (38). As in other cancers, loss of GPX3 expression may also have tumor suppressor functions in melanoma carcinogenesis and progression via failures in the cellular antioxidant system.

The epigenetic inactivation of GPX3 may be a crucial mechanism in the pathogenesis of melanoma, and GPX3 may serve as a possible predictive or prognostic biomarker and therapeutic target for melanoma patients.

\section{Acknowledgements}

This work was supported by National Natural Science Foundation of China (NSFC, no. 81560503) and Basic Science Research Program through the National Research Foundation of Korea (NRF) funded by the Ministry of Education, Science and Technology (2011-0022376).

\section{References}

1. Dreher D and Junod AF: Role of oxygen free radicals in cancer development. Eur J Cancer 32A: 30-38, 1996.

2. Cooke MS, Evans MD, Dizdaroglu M and Lunec J: Oxidative DNA damage: Mechanisms, mutation, and disease. FASEB J 17: $1195-1214,2003$.

3. Malins DC, Polissar NL and Gunselman SJ: Progression of human breast cancers to the metastatic state is linked to hydroxyl radicalinduced DNA damage. Proc Natl Acad Sci USA 93: 2557-2563, 1996.

4. Cerutti PA: Prooxidant states and tumor promotion. Science 227 375-381, 1985.

5. Sies H: Oxidative stress: Oxidants and antioxidants. Exp Physiol 82: 291-295, 1997.

6. Dröge W: Free radicals in the physiological control of cell function. Physiol Rev 82: 47-95, 2002.

7. Tham DM, Whitin JC, Kim KK,Zhu SX and Cohen HJ: Expression of extracellular glutathione peroxidase in human and mouse gastrointestinal tract. Am J Physiol 275: G1463-G1471, 1998.

8. Björnstedt M, Xue J, Huang W, Akesson B and Holmgren A: The thioredoxin and glutaredoxin systems are efficient electron donors to human plasma glutathione peroxidase. J Biol Chem 269: 2938229384, 1994.

9. Yamamoto Y and Takahashi K: Glutathione peroxidase isolated from plasma reduces phospholipid hydroperoxides. Arch Biochem Biophys 305: 541-545, 1993.

10. Esworthy RS, Chu FF, Paxton RJ, Akman S and Doroshow JH: Characterization and partial amino acid sequence of human plasma glutathione peroxidase. Arch Biochem Biophys 286 330-336, 1991.

11. Brigelius-Flohé $\mathrm{R}$ and Kipp A: Glutathione peroxidases in different stages of carcinogenesis. Biochim Biophys Acta 1790: $1555-1568,2009$
12. Yoshimura S, Suemizu H, Taniguchi Y, Watanabe K, Nomoto Y, Katsuoka Y, Arimori S and Moriuchi T: Molecular cloning of human plasma glutathione peroxidase gene and its expression in the kidney. Nucleic Acids Symp Ser (25): 163-164, 1991.

13. Yoshimura S, Suemizu H, Taniguchi Y, Arimori K, Kawabe N and Moriuchi T: The human plasma glutathione peroxidaseencoding gene: Organization, sequence and localization to chromosome 5q32. Gene 145: 293-297, 1994.

14. Takebe G, Yarimizu J, Saito Y, Hayashi T, Nakamura H, Yodoi J, Nagasawa $S$ and Takahashi K: A comparative study on the hydroperoxide and thiol specificity of the glutathione peroxidase family and selenoprotein P. J Biol Chem 277: 41254-41258, 2002.

15. Comhair SA and Erzurum SC: The regulation and role of extracellular glutathione peroxidase. Antioxid Redox Signal 7: 72-79, 2005.

16. Baker RD, Baker SS, LaRosa K, Whitney C and Newburger PE: Selenium regulation of glutathione peroxidase in human hepatoma cell line Hep3B. Arch Biochem Biophys 304: 53-57, 1993.

17. Clark LC, Combs GF Jr, Turnbull BW, Slate EH, Chalker DK, Chow J, Davis LS, Glover RA, Graham GF, Gross EG, et al; Nutritional Prevention of Cancer Study Group: Effects of selenium supplementation for cancer prevention in patients with carcinoma of the skin. A randomized controlled trial. JAMA 276: 1957-1963, 1996.

18. Avissar N, Ornt DB, Yagil Y, Horowitz S, Watkins RH, Kerl EA, Takahashi K, Palmer IS and Cohen HJ: Human kidney proximal tubules are the main source of plasma glutathione peroxidase. Am J Physiol 266: C367-C375, 1994

19. Schomburg L and Köhrle J: On the importance of selenium and iodine metabolism for thyroid hormone biosynthesis and human health. Mol Nutr Food Res 52: 1235-1246, 2008.

20. Köhrle J: Selenium and the control of thyroid hormone metabolism. Thyroid 15: 841-853, 2005.

21. Lapointe J, Kimmins S, Maclaren LA and Bilodeau JF: Estrogen selectively up-regulates the phospholipid hydroperoxide glutathione peroxidase in the oviducts. Endocrinology 146: 2583-2592, 2005 .

22. Hoffenberg EJ, Deutsch J, Smith S and Sokol RJ: Circulating antioxidant concentrations in children with inflammatory bowel disease. Am J Clin Nutr 65: 1482-1488, 1997.

23. Comhair SA, Bhathena PR, Farver C, Thunnissen FB and Erzurum SC: Extracellular glutathione peroxidase induction in asthmatic lungs: Evidence for redox regulation of expression in human airway epithelial cells. FASEB J 15: 70-78, 2001.

24. Tham DM, Whitin JC and Cohen HJ: Increased expression of extracellular glutathione peroxidase in mice with dextran sodium sulfate-induced experimental colitis. Pediatr Res 51: 641-646, 2002.

25. Iwata $K$, Nishinaka $T$, Matsuno $K$ and Yabe-Nishimura $C$ : Increased gene expression of glutathione peroxidase-3 in diabetic mouse heart. Biol Pharm Bull 29: 1042-1045, 2006.

26. Mörk H, Lex B, Scheurlen M, Dreher I, Schütze N, Köhrle J and Jakob F: Expression pattern of gastrointestinal selenoproteins - targets for selenium supplementation. Nutr Cancer 32: 64-70, 1998.

27. Yu YP, Yu G, Tseng G, Cieply K, Nelson J, Defrances M, Zarnegar R, Michalopoulos G and Luo JH: Glutathione peroxidase 3 , deleted or methylated in prostate cancer, suppresses prostate cancer growth and metastasis. Cancer Res 67: 8043-8050, 2007.

28. Jemal A, Thomas A, Murray T and Thun M: Cancer statistics, 2002. CA Cancer J Clin 52: 23-47, 2002.

29. Zhang X, Zheng Z, Yingji S, Kim H, Jin R, Renshu L, Lee DY, Roh MR and Yang S: Downregulation of glutathione peroxidase 3 is associated with lymph node metastasis and prognosis in cervical cancer. Oncol Rep 31: 2587-2592, 2014.

30. Luo X, Wei B, Chen A, Zhao H, Huang $\mathrm{K}$ and Chen J: Methylation-mediated loss of SFRP2 enhances melanoma cell invasion via Wnt signaling. Am J Transl Res 8: 1502-1509, 2016.

31. El Haddad M, Jean E, Turki A, Hugon G, Vernus B, Bonnieu A, Passerieux E, Hamade A, Mercier J, Laoudj-Chenivesse D, et al: Glutathione peroxidase 3, a new retinoid target gene, is crucial for human skeletal muscle precursor cell survival. J Cell Sci 125: 6147-6156, 2012

32. Barrett CW, Ning W, Chen X, Smith JJ, Washington MK, Hill KE, Coburn LA, Peek RM, Chaturvedi R, Wilson KT, et al: Tumor suppressor function of the plasma glutathione peroxidase gpx3 in colitis-associated carcinoma. Cancer Res 73: 1245-1255, 2013. 
33. Cameron EE, Bachman KE, Myöhänen S, Herman JG and Baylin SB: Synergy of demethylation and histone deacetylase inhibition in the re-expression of genes silenced in cancer. Nat Genet 21: 103-107, 1999.

34. Peng DF, Hu TL, Schneider BG, Chen Z, Xu ZK and El-Rifai W: Silencing of glutathione peroxidase 3 through DNA hypermethylation is associated with lymph node metastasis in gastric carcinomas. PLoS One 7: e46214, 2012.

35. Zhang X, Yang JJ, Kim YS, Kim KY, Ahn WS and Yang S: An 8-gene signature, including methylated and down-regulated glutathione peroxidase 3 , of gastric cancer. Int J Oncol 36: 405-414, 2010.
36. Yang ZL, Yang L, Zou Q, Yuan Y, Li J, Liang L, Zeng G and Chen S: Positive ALDH1A3 and negative GPX3 expressions are biomarkers for poor prognosis of gallbladder cancer. Dis Markers 35: 163-172, 2013.

37. Kaiser MF, Johnson DC, Wu P, Walker BA, Brioli A, Mirabella F, Wardell CP, Melchor L, Davies FE and Morgan GJ: Global methylation analysis identifies prognostically important epigenetically inactivated tumor suppressor genes in multiple myeloma. Blood 122: 219-226, 2013.

38. Ouyang X, DeWeese TL, Nelson WG and Abate-Shen C: Lossof-function of Nkx3.1 promotes increased oxidative damage in prostate carcinogenesis. Cancer Res 65: 6773-6779, 2005. 